\title{
Remarks on the Surface Area and Equality Conditions in Regular Forms Part IV: Pyramidal Forms
}

\author{
Ahmed A. Elkhateeb • Esraa A. Elkhateeb
}

Published online: 9 September 2014

(C) Kim Williams Books, Turin 2014

\begin{abstract}
Following the same methodology and rules that were applied in the previous Parts I-III of this work, Part IV presents the mathematical remarks on the right regular pyramidal forms, either complete or truncated (a frustum of pyramid). The first remark examines the effects of $\theta$ and $\beta$ on $S$. The second remark calculates the minimum total surface area $\left(\mathrm{S}_{\mathrm{Min}}\right)$ in two cases, case of constant $\theta$ (variable $\beta$ ) and case of variable $\theta$ (constant $\beta$ ). The third remark calculates walls ratio $R_{W}$ and the critical walls ratio $\boldsymbol{R}_{\boldsymbol{W}}$. The last remark calculates the numerical equality between $\mathrm{S}$ and $\mathrm{V}$. In conclusion, the importance of the findings of the entire work (Parts I-IV) in advanced building analysis is highlighted and discussed.
\end{abstract}

Keywords Acoustics · Pyramids · Pyramidal forms · Surface area · Walls ratio $\cdot$ Building analysis $\cdot$ Minimum total surface area

Numerical equality

\section{Introduction}

Pyramidal forms are some of the oldest forms that humans have ever known. Historically, pyramids were constructed in different compositions (complete, truncated and stepped) in many places of ancient world, such as Egypt, Mexico and Latin America. The step pyramid of Djoser (Zoser, in the Saqqara necropolis) may be the first of this type (built around 2800 BC). In the realm of modern architecture,

\footnotetext{
A. A. Elkhateeb ( ()

Department of Architecture, Faculty of Environmental Designs, King Abdulaziz University, Jeddah, Saudi Arabia

e-mail: aelkhateeb@kau.edu.sa

E. A. Elkhateeb

Physics Department, Faculty of Science, Ain Shams University, Cairo, Egypt

e-mail: dr.esraaelkhateeb@gmail.com
} 
the pyramidal form still attracts architects. There are several examples for modern pyramidal buildings in different places around the world, for example the Unknown Soldier Memorial in Cairo, by Sami Rafi (1973), Louvre pyramid in Paris, by I. M. Pei (1989) and Luxor Hotel, Las Vegas, by Veldon Simpson (1993).

This Part IV applies the same methodology and assumptions that were established in the previous Parts I-III (Elkhateeb 2014a, b; Elkhateeb and Elkhateeb 2014) of this work to investigate the case of the right regular pyramid (either complete or truncated) so as to answer the following questions:

- How do the angles $\theta$ and $\beta$ affect $S$ ?

- When does $\mathrm{S}$ become minimum $\left(\mathrm{S}_{\mathrm{Min}}\right)$ ?

- What is the ratio between walls total surface area $S_{\mathrm{W}}$ and $\mathrm{S}\left(\mathrm{S}_{\mathrm{W}} / \mathrm{S}=\mathrm{R}_{\mathrm{W}}\right)$ ?

- When $\mathrm{S}$ equals V?

\section{Notations}

In this work, the following terms mean:

Ar Base area, area of the lower base (truncated pyramid) $\left(\mathrm{m}^{2}\right)$

$\mathrm{Ar}_{\mathrm{s}} \quad$ Area of one side face of the pyramid $\left(\mathrm{m}^{2}\right)$

$\mathrm{Ar}_{\mathrm{U}}$ Area of the upper base (truncated pyramid) $\left(\mathrm{m}^{2}\right)$

$\boldsymbol{\beta}_{\boldsymbol{o}} \quad$ The critical dihedral angle, the angle at which $\mathrm{S}$ of a complete right regular pyramid becomes minimum

$\mathrm{h} \quad$ The altitude of the triangle $(\mathrm{m})$

$\mathrm{h}_{\mathrm{s}} \quad$ Slant height $(\mathrm{m})$

$\mathrm{H}_{\mathrm{R}} \quad$ The altitude of the pyramid (m)

$\boldsymbol{H}_{\boldsymbol{R} o}$ The critical room height, the height that fulfills $(\mathrm{S}-\mathrm{V})$ equality (m)

$\mathrm{n} \quad$ Number of sides

Per Perimeter (m)

S Room total surface area $\left(\mathrm{m}^{2}\right)$

$\mathrm{S}_{\text {Min }}$ The minimum total surface area $\left(\mathrm{m}^{2}\right)$

$\mathrm{S}_{\mathrm{W}} \quad$ Walls total surface area $\left(\mathrm{m}^{2}\right)$

$\mathrm{r} \quad$ Radius (m)

$\boldsymbol{r}_{\boldsymbol{o}} \quad$ The critical radius, the radius that fulfills (Per - Ar) equality (m)

$\mathrm{R}_{\mathrm{W}} \quad$ Walls ratio, $\mathrm{S}_{\mathrm{W}} / \mathrm{S}$ (ratio)

$\boldsymbol{R}_{\boldsymbol{W}}$ The critical walls ratio, the ratio between walls total surface area and total surface area when $\mathrm{S}$ is minimum $\left(\mathrm{S}_{\mathrm{Min}}\right)$ (ratio)

$\mathrm{V} \quad$ Room volume $\left(\mathrm{m}^{3}\right)$

$\omega_{o}$ The critical ratio, the ratio between $\mathrm{H}_{\mathrm{R}}$ and $\mathrm{r}$ when $\mathrm{S}$ is minimum $\left(\mathrm{S}_{\mathrm{Min}}\right)$ (ratio)

The other terms will be illustrated in figures according to each case as required.

\section{Right Regular Pyramid}

A right regular pyramid is a pyramid that has its apex aligned directly above the center of its regular base. This base can be any regular polygon (a multi-sided shape) from the equilateral triangle $(n=3)$ to the circle $(n=\infty)$ in which: 
- All sides are congruent.

- All angles are congruent (thus, the angle $\psi$ is constant and equal to $360 / \mathrm{n}$ ).

Through this part, it is assumed that $\mathrm{V}, \theta$ and $\beta$ are the independent variables whereas Ar, Per, and $\mathrm{S}$ are the dependent ones. Figure 1 illustrates the different variables: $\theta, \psi, h, r, L, \beta, H_{R}$ and $h_{s}$ in a right regular pyramid.

The Mathematical Relationships of Right Regular Pyramids

In Part III of this work, the main mathematical functions between $\theta, \mathrm{L}, \mathrm{h}, \mathrm{r}$, Per and Ar have been driven. These functions are also applied in this part as long as the base of the pyramid is regular as defined and assumed. While this section will not repeat those functions of Part III, it will derive additional mathematical functions among $r$, $\mathrm{S}, \beta$ and $\mathrm{V}$ (see Fig. 1). These new functions will be utilized later to determine $\mathrm{S}_{\mathrm{Min}}$ and calculate the equality conditions.

A right regular pyramid can be fully identified when its volume $\mathrm{V}$, number of sides $\mathrm{n}$ (thus $\theta$ ) and the dihedral angle $\beta$ are known. Given these variables, it can be proved that (see Part III):

$$
r=\sqrt[3]{\frac{3 V}{n \sin \theta \cos ^{2} \theta \tan \beta}} .
$$

This equation yields the radius of a circle that contains the regular base of a pyramid knowing $\mathrm{n}, \mathrm{V}$ and $\beta$. In addition, it also relates $\mathrm{r}$ to the dihedral angle $\beta$ for a given $\mathrm{V}$. Given $\mathrm{r}$, both $\mathrm{H}_{\mathrm{R}}$ and $\mathrm{Ar}$ can be calculated as:

$$
H_{R}=r \cos \theta \tan \beta
$$

and (Gieck and Gieck 2006)

$$
A r=\frac{3 V}{H_{R}} .
$$

From Eq. 3, $\mathrm{H}_{\mathrm{R}}$ can be also calculated as:

$$
H_{R}=\frac{3 V}{n r^{2} \sin \theta \cos \theta} .
$$

In such pyramid, there are two possibilities for modifying its dimensions assuming that $\mathrm{V}$ is constant:

- In the first, $\beta$ and Ar are variables whereas $n$ (thus $\theta$ ) is constant.

- In the second, $\beta$ is constant whereas $n$ and $\operatorname{Ar}$ (i.e., $r$ ) are variables.

As can be concluded from the previous options, Ar is always variable as long as both $\mathrm{V}$ and $\beta$ were determined. From the first principles, the slant height $\mathrm{h}_{\mathrm{S}}$ can be calculated as:

$$
h_{S}=\frac{H_{R}}{\sin \beta} .
$$

The area of one face of this pyramid $\mathrm{Ar}_{\mathrm{S}}$ can be calculated from: 

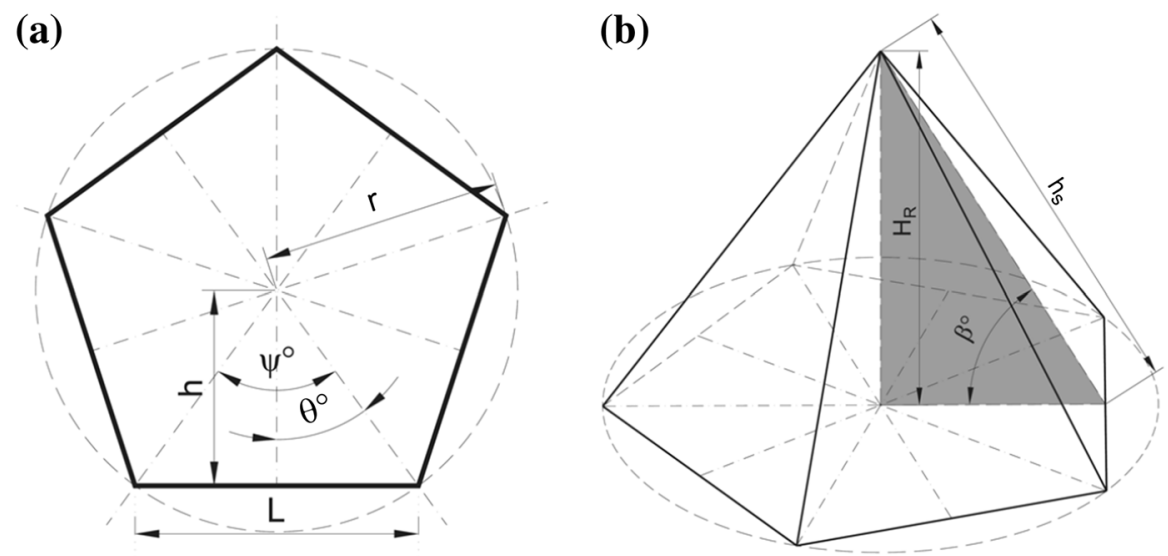

Fig. 1 Right regular pyramids, the different variables: a left a regular multi-sided base; b right a pyramidal form. Image: author

$$
A r_{S}=\frac{L H_{R}}{2 \sin \beta}
$$

The total surface area of a right regular pyramid can be calculated as:

$$
S=\frac{n r H_{R} \sin \theta}{\sin \beta}+n r^{2} \sin \theta \cos \theta .
$$

By substitution for $\mathrm{H}_{\mathrm{R}}$ according to Eq. 4, Eq. 7 can be rewritten as:

$$
S=\frac{3 V}{r \cos \theta \sin \beta}+n r^{2} \sin \theta \cos \theta .
$$

If $\mathrm{r}$ is replaced by its equivalent value according to Eq. 1, Eq. 8 can be rewritten as:

$$
S=\frac{3 V}{\left(\sqrt[3]{\frac{3 V}{n \sin \theta \cos ^{2} \theta \tan \beta}}\right) \times \cos \theta \sin \beta}+\left(\frac{3 V}{n \sin \theta \cos ^{2} \theta \tan \beta}\right)^{\frac{2}{3}} \times n \sin \theta \cos \theta .
$$

Given that $\mathrm{n}=180 / \theta$, Eq. 9 will be:

$$
\begin{aligned}
S= & \frac{3 V}{\left(\sqrt[3]{\frac{3 V}{(180 / \theta) \sin \theta \cos ^{2} \theta \tan \beta}}\right) \times \cos \theta \sin \beta} \\
& +\left(\frac{3 V}{(180 / \theta) \sin \theta \cos ^{2} \theta \tan \beta}\right)^{\frac{2}{3}} \times\left(\frac{180}{\theta}\right) \sin \theta \cos \theta .
\end{aligned}
$$

Remark 1: Effects of $\theta$ and $\beta$ on $S$

The effect of $\theta$ on $S$ in the right regular pyramid can be concluded from Eq. 7 when $\beta$ is constant (Fig. 2). This effect resembles the case of the regular multi-sided right prisms (see Part III). In summary, $\mathrm{S}$ is an increasing function of $\theta$ (thus a decreasing function of $n)$. This means that $S$ of a right regular triangular pyramid $(n=3)$ is 


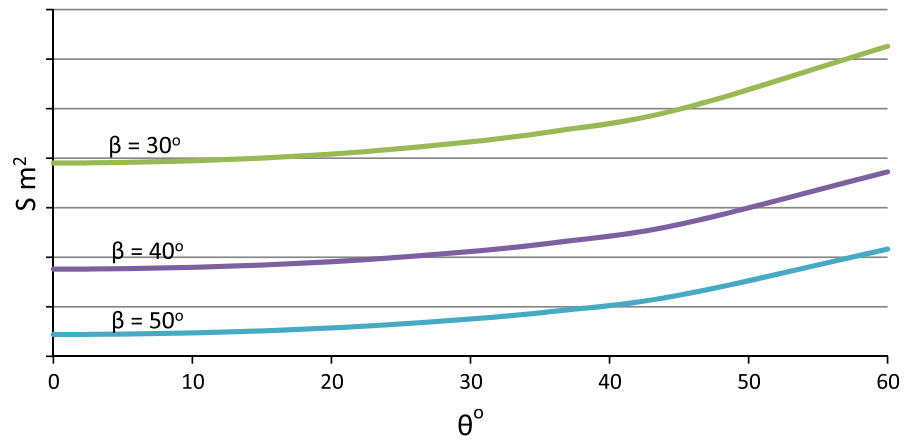

Fig. 2 Effect of $\theta$ on $S$ according to Eq. 7 (different values of $\beta$ )

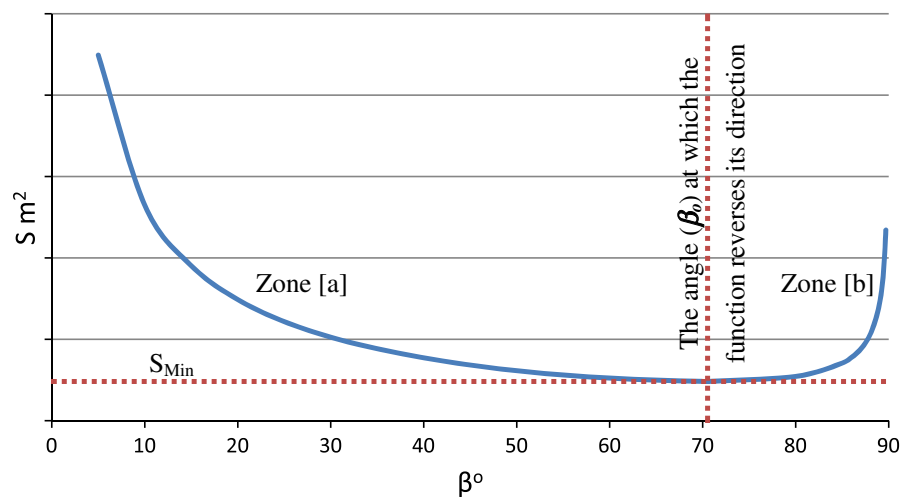

Fig. 3 Effect of $\beta$ on $S$ according to Eq. 7 (case of $n=3$ )

larger than a right cone $(\mathrm{n}=\infty)$ provided that both have the same $\mathrm{V}$ (Elkhateeb 2014b).

Similarly, the effect of $\beta$ on $S$ can be also concluded from Eq. 7 when $\theta$ is constant. Figure 3 illustrates this case graphically. It is clear from this figure that the function is similar to the case of the regular right prisms (see for example Part III) where the function changes its direction at a certain angle. This angle will be called $\boldsymbol{\beta}_{\boldsymbol{o}}$ (see "Case I: Variable $\beta$ and Constant $\theta$ "). $\boldsymbol{\beta}_{\boldsymbol{o}}$ splits the function into two main zones:

- Zone [a]: where $0^{\circ}<\beta<\boldsymbol{\beta}_{\boldsymbol{o}}$. In this zone $S$ is a decreasing function of $\beta$.

- Zone [b]: where $\boldsymbol{\beta}_{\boldsymbol{o}}<\beta<90^{\circ}$. In this zone $\mathrm{S}$ is an increasing function of $\beta$.

Remark 2: The Minimum Total Surface Area, $\mathrm{S}_{\text {Min }}$

Following the same approach that was applied previously in the earlier parts of this work and as mentioned in "The Mathematical Relationships of Right Regular Pyramids", two cases will be considered: 
Table 1 Values of $\omega_{o}$ for the common right regular pyramids according to their bases

\begin{tabular}{lllllll}
\hline $\boldsymbol{\theta}^{\mathbf{o}}$ & 60 & 45 & 36 & 30 & 22.5 & $\mathbf{0}$ \\
\hline Shape of base & Triangle & Square & Pentagon & Hexagon & Octagon & Circle \\
$\boldsymbol{\omega}_{\boldsymbol{o}}$ & 1.4142 & 2.00 & 2.288 & 2.449 & 2.613 & 2.828
\end{tabular}

- Case of variable $\beta$ and constant $\theta$;

- Case of constant $\beta$ and variable $\theta$.

\section{Case I: Variable $\beta$ and Constant $\theta$}

In this case, among the different right regular pyramids that have the same $\theta$ and $\mathrm{V}$, $\mathrm{S}_{\mathrm{Min}}$ occurs when the first derivative of Eq. 9 equals zero

$$
\begin{aligned}
\frac{d s}{d \beta}= & \frac{1}{3} \frac{3^{2 / 3} V^{2}\left(1+\tan ^{2} \beta\right)}{\left(\frac{V}{n \sin \theta \cos ^{2} \theta \tan \beta}\right)^{4 / 3} \cos ^{3} \theta \sin \theta \sin \beta \tan ^{2} \beta}-\frac{3^{2 / 3} V \cos \beta}{\left(\frac{V}{n \sin \theta \cos ^{2} \theta \tan \beta}\right)^{1 / 3} \cos \theta \sin ^{2} \beta} \\
& -\frac{2}{3} \frac{3^{2 / 3} V\left(1+\tan ^{2} \beta\right)}{\left(\frac{V}{n \sin \theta \cos ^{2} \theta \tan \beta}\right)^{1 / 3} \cos \theta \tan ^{2} \beta}=0 .
\end{aligned}
$$

The solution of Eq. 11 yields the value of $\boldsymbol{\beta}_{\boldsymbol{o}}$, the dihedral angle at which $\mathrm{S}$ reaches its minimum value. As can be seen, this last equation is very complex to solve analytically in order to get the value of $\boldsymbol{\beta}_{\boldsymbol{o}}$. Possible solutions for such problem are numerical calculations or graphical representation for the function $\boldsymbol{d} \boldsymbol{S} / \boldsymbol{d} \boldsymbol{\beta}$. In our case, the program Maple ${ }^{\circledR}$ for symbolic calculations was utilized to get the value of $\boldsymbol{\beta}_{\boldsymbol{o}}$. The calculations lead to:

$$
\beta_{o}=\arctan (2 \sqrt{2}), \quad \text { i.e. } \beta_{o}=70.528779^{\circ} .
$$

Thus, a right regular pyramid that has a dihedral angle equal to $\boldsymbol{\beta}_{\boldsymbol{o}}$ possesses the minimum total surface area among others that have the same $\theta$ and $\mathrm{V}$. In the right regular pyramids, the critical ratio $\omega_{o}$ can be calculated as:

$$
\omega_{o}=2 \sqrt{2} \cos \theta \text {. }
$$

It can be concluded from Eq. 13 that $\omega_{o}$ also depends on $\theta$ and is an increasing function of $\theta$ (thus a decreasing function of $n$ ). Table 1 lists the values of $\omega_{o}$ for the most common right regular pyramids according to their bases. $\omega_{o}$ can be utilized as an alternate way to calculate $S_{\text {Min }}$ for a right regular pyramid when its $\mathrm{V}, \theta$ and $\beta$ are known by applying Eq. 1 to calculate r, then Eq. 13 to get $\mathrm{H}_{\mathrm{R}}$. The other variables can be calculated using the appropriate formulas (see "The Mathematical Relationships of Right Regular Pyramids" here and Part III (Elkhateeb 2014b).

Similar to the cases of triangular and quadratic right prisms, the two relationships $\left(\mathrm{H}_{\mathrm{R}}-\mathrm{S}\right)$ and $(\mathrm{Ar}-\mathrm{S})$ depend on $\boldsymbol{\beta}_{\boldsymbol{o}}$, which divides the functions into two zones (see Figs. 4, 5):

- Zone [a]: where $\boldsymbol{\beta}<\boldsymbol{\beta}_{\boldsymbol{o}}$. In this zone, $\mathrm{S}$ is a decreasing function of $\mathrm{H}_{\mathrm{R}}$ (see Fig. 4) and an increasing function of Ar (see Fig. 5). Note that the location of 


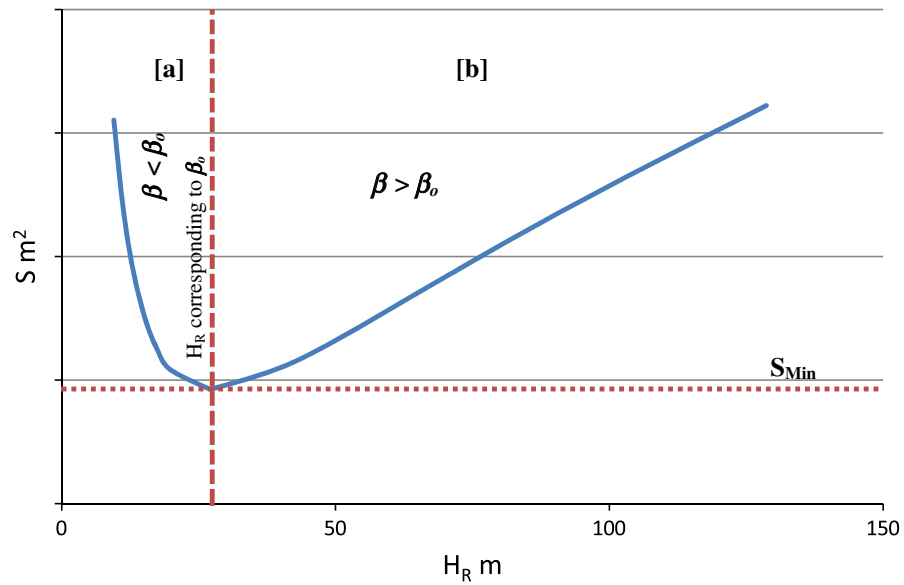

Fig. 4 The relationship of $H_{R}$ to $S$ (case of triangle, $n=3$ )

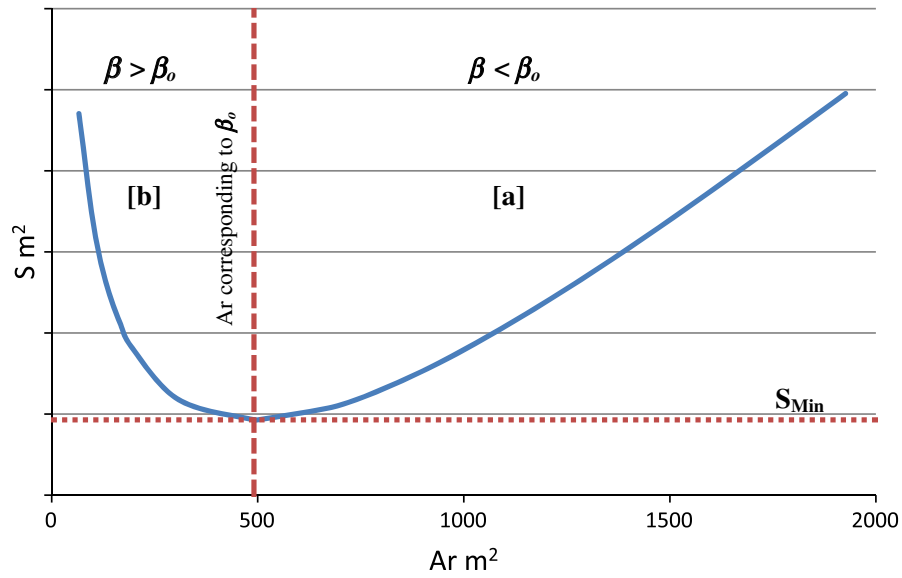

Fig. 5 The relationship of Ar to $S$ (case of pentagon, $n=5$ )

the zones is reversed in Fig. 5, thus any increase in pyramid height will decrease its total surface area.

- Zone [b]: where $\boldsymbol{\beta}>\boldsymbol{\beta}_{\boldsymbol{o}}$. In this zone, $\mathrm{S}$ is an increasing function of $\mathrm{H}_{\mathrm{R}}$ and a decreasing function of Ar. This means that an increase in $H_{R}$ will increase $S$.

\section{Case II: Constant $\beta$ and Variable $\theta$}

In this case, among the different right regular pyramids that have the same $\beta$ and $\mathrm{V}$, $\mathrm{S}_{\mathrm{Min}}$ occurs when the first derivative of Eq. 10 equals zero. Thus, 


$$
\begin{aligned}
\frac{d s}{d \theta}= & -\frac{1}{3} \frac{3^{2 / 3} V^{2}\left(\frac{1}{180 \sin \theta \cos ^{2} \theta \tan \beta}-\frac{\theta}{180 \sin ^{2} \theta \cos \theta \tan \beta}+\frac{2 \theta}{180 \cos ^{3} \theta \tan \beta}\right)}{\left(\frac{V \theta}{180 \sin \theta \cos ^{2} \theta \tan \beta}\right)^{4 / 3} \cos \theta \sin \beta} \\
& +\frac{3^{2 / 3} V \sin \theta}{\left(\frac{V \theta}{180 \sin \theta \cos ^{2} \theta \tan \beta}\right)^{1 / 3} \cos ^{2} \theta \sin \beta} \\
& +\frac{2}{3} \frac{3^{2 / 3} \times 180 V \cos \theta \sin \theta\left(\frac{1}{180 \sin \theta \cos ^{2} \theta \tan \beta}-\frac{\theta}{180 \sin ^{2} \theta \cos \theta \tan \beta}+\frac{2 \theta}{180 \cos ^{3} \theta \tan \beta}\right)}{\left(\frac{V \theta}{180 \sin \theta \cos ^{2} \theta \tan \beta}\right)^{4 / 3} \theta} \\
& -\frac{1}{\theta^{2}}\left(\frac{3 V \theta}{180 \sin \theta \cos ^{2} \theta \tan \beta}\right)^{2 / 3} 180 \sin \theta \cos \theta+\frac{180}{\theta}\left(\frac{3 V \theta}{180 \sin \theta \cos ^{2} \theta \tan \beta}\right) \\
& \times\left(\cos ^{2} \theta-\sin { }^{2} \theta\right)=0 .
\end{aligned}
$$

Again, due to the complexity of this last equation, the program Maple ${ }^{\circledR}$ for symbolic calculations was utilized to get the value of $\boldsymbol{\theta}$ at which $\mathrm{S}$ becomes minimum. The calculations indicate that $S_{\text {Min }}$ occurs when $\theta \rightarrow 0$.

Thus, among the different right regular pyramids, a cone has the minimum total surface area. This conclusion completely agrees with the numerical solution presented in Fig. 2 and discussed in "Remark 1: Effects of $\theta$ and $\beta$ on $S$ ".

\section{Remark 3: Walls Ratio $\mathrm{R}_{\mathrm{W}}$}

In right regular pyramids, $\mathrm{R}_{\mathrm{W}}$ can be mathematically defined as:

$$
R_{W}=\frac{n \times A r_{S}}{A r+n \times A r_{S}} .
$$

By substitution for $\mathrm{Ar}_{\mathrm{S}}$ according to Eq. 6 and $\mathrm{Ar}$ (see Part III), Eq. 15 can be rewritten as:

$$
R_{W}=\frac{H_{R}}{H_{R}+r \cos \theta \sin \beta} .
$$

If $\mathrm{H}_{\mathrm{R}}$ is replaced by its equivalent value according to Eq. 2, then Eq. 16 will be:

$$
R_{W}=\frac{\tan \beta}{\tan \beta+\sin \beta} .
$$

According to Eq. 17, $\mathrm{R}_{\mathrm{W}}$ depends completely on $\beta$ regardless the values of $\mathrm{V}$ and $\theta$. For example, $\mathrm{R}_{\mathrm{W}}=0.586$ for $\beta=45^{\circ}$. Figure 6 represents the relationship $\beta-$ $\mathrm{R}_{\mathrm{W}}$, it is clear that $\mathrm{R}_{\mathrm{W}}$ is an increasing function of $\beta$. According to Eq. 17, it can be concluded that $\boldsymbol{R}_{\boldsymbol{W o}}=0.75$.

Remark 4: Case of Numerical Equality

In Part III (Elkhateeb 2014b), the numerical equality between Ar and Per was calculated. As the same condition applies here, it is not repeated again. Thus, this 


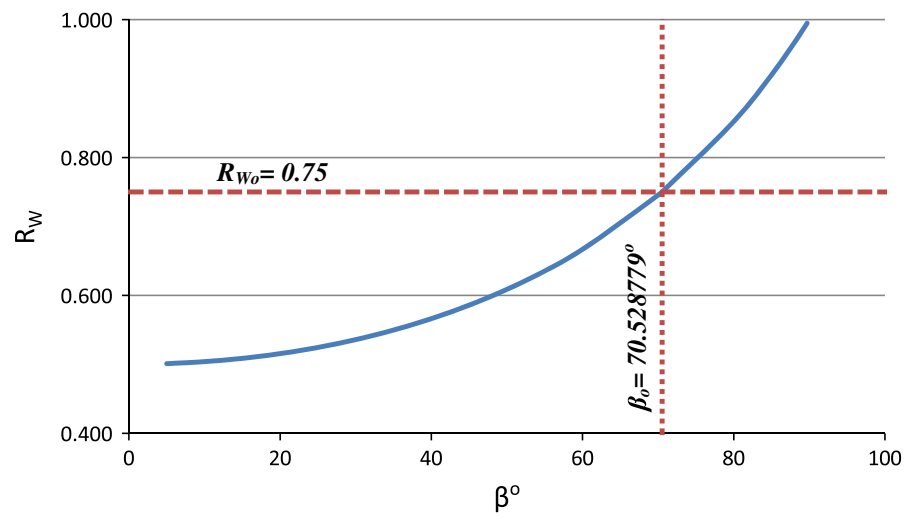

Fig. 6 The ratio $R_{W}$ as a function of $\beta$

section considers only the numerical equality between $\mathrm{S}$ and $\mathrm{V}$ in right regular pyramids.

\section{Equality of $S$ and $V$}

According to Part III, Eqs. 2 and 7, this numerical equality occurs when:

$$
\frac{n r H_{R} \sin \theta}{\sin \beta}+n r^{2} \sin \theta \cos \theta=\frac{1}{3} H_{R} n r^{2} \sin \theta \cos \theta .
$$

By applying the principles of algebra, Eq. 18 will be:

$$
H_{R o}=\frac{3 r \cos \theta \sin \beta}{r \cos \theta \sin \beta-3} \text {. }
$$

Thus, in right regular pyramids with given $\theta, \beta$ and $\mathrm{Ar}$, the numerical equality between $\mathrm{S}$ and V occurs only if Eq. 19 has been satisfied. This can be calculated in the following sequence:

- Determine $\theta$ (or $\mathrm{n}$ ), $\beta$ and Ar;

- Apply Eq. 4 (Part III) to get r;

- Substitute in Eq. 19 to get the critical room height $\boldsymbol{H}_{\boldsymbol{R} \boldsymbol{o}}$ (see "Notations") that fulfills this equality.

The relationship $\mathrm{Ar}-\boldsymbol{H}_{\boldsymbol{R} \boldsymbol{o}}$ resembles the previous cases studied in this work. The minus sign $(-)$ in the denominator of Eq. 19 indicates the limit under which this equality will never exist. Mathematically, this equality will never exist if:

$$
r \cos \theta \sin \beta \leq 3 \text {. }
$$

In the special case where $n \rightarrow \infty$ (i.e., a cone), $\theta \rightarrow 0$. As $\cos \mathbf{0}=1$, thus, Eq. 19 will be:

$$
H_{R o}=\frac{3 r \sin \beta}{r \sin \beta-3} .
$$




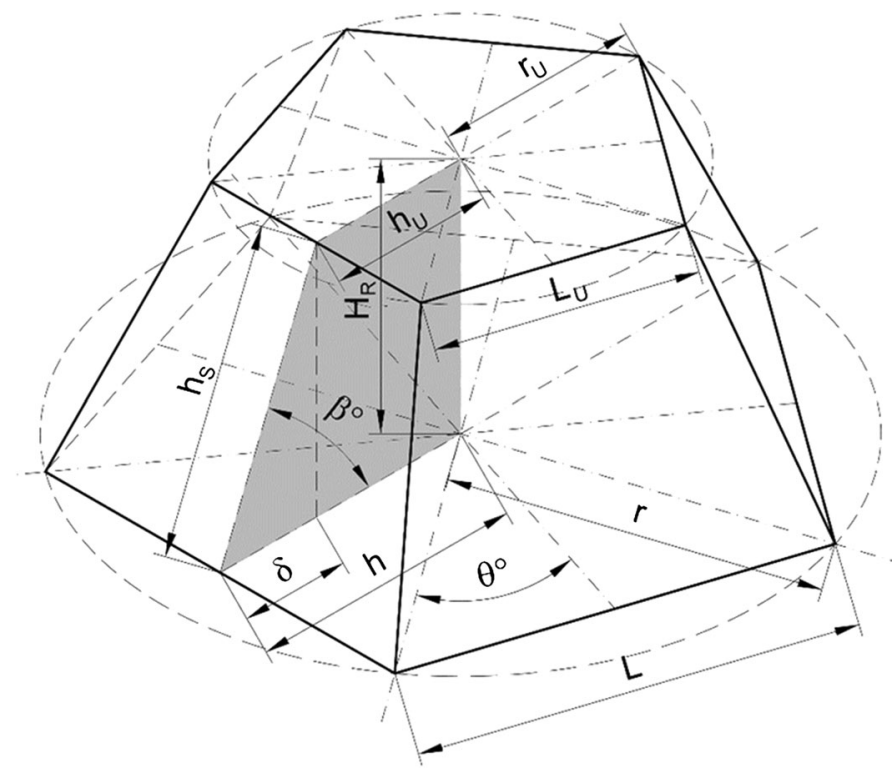

Fig. 7 Truncated right regular pyramid, different variables, see also Fig. 1 for the other variables

\section{Truncated Right Regular Pyramid}

The truncated right regular pyramid is a portion of a right regular pyramid included between two parallel bases. In such a pyramid:

- The slant height $h_{S}$ is the altitude of a side face.

- The lateral edges are equal, and the side faces are equal isosceles trapezoids.

- The two bases are similar parallel regular polygons.

In addition to the variables $\mathrm{V}, \theta$ and $\beta$ which identified the complete right regular pyramid, the height $\mathrm{H}_{\mathrm{R}}$ (see Fig. 7) must be also identified in case of a frustum of a pyramid. Thus, in the following section, it is assumed that $\mathrm{V}, \theta, \beta$ and $\mathrm{H}_{\mathrm{R}}$ are the independent variables whereas Ar, Per, and $S$ are the dependent ones. Figure 7 illustrates the variables: $\theta, \beta, \mathrm{r}, \mathrm{r}_{\mathrm{U}}, \mathrm{L}, \mathrm{L}_{\mathrm{U}}, \mathrm{H}_{\mathrm{R}}, \delta$ and $\mathrm{h}_{\mathrm{S}}$.

The Mathematical Relationships of Truncated Right Regular Pyramids

Beside the main mathematical relationships previously listed in Part III and "The Mathematical Relationships of Right Regular Pyramids" of this part, this section lists additional mathematical formulas regarding the truncated right regular pyramid. From the first principles, it can be concluded that:

$$
\delta=\frac{H_{R}}{\tan \beta}
$$


and

$$
h_{U}=h-\delta .
$$

By replacing $\mathrm{h}, \mathrm{h}_{\mathrm{U}}$ and $\delta$ with their equivalent values (see Part III, Elkhateeb 2014b), then Eq. 23 will be:

$$
r_{U}=r-\frac{H_{R}}{\cos \theta \tan \beta} .
$$

From the first principles again, the total surface area $\mathrm{S}$ of a truncated right regular pyramid can be calculated as:

$$
S=n h_{S}\left(\frac{L+L_{U}}{2}\right)+A r+A r_{U}
$$

and the volume of such pyramid can be calculated from

$$
V=\frac{1}{3} H_{R}\left(A r+A r_{U}+\sqrt{A r \cdot A r_{U}}\right)
$$

(Gieck and Gieck 2006).

If $\mathrm{Ar}$ and $\mathrm{Ar}_{\mathrm{U}}$ are replaced by their equivalent values (see Part III, Elkhateeb 2014b), then Eq. 26 will be:

$$
V=\frac{1}{3} n H_{R} \sin \theta \cos \theta\left(r^{2}+r_{U}^{2}+r \cdot r_{U}\right) .
$$

By substitution for $r_{U}$ according to Eq. 24, Eq. 27 will be:

$$
V=\frac{1}{3} H_{R} \sin \theta \cos \theta\left[r^{2}+\left(r-\frac{H_{R}}{\cos \theta \tan \beta}\right)^{2}+r \cdot\left(r-\frac{H_{R}}{\cos \theta \tan \beta}\right)\right] .
$$

This leads to a quadratic equation with one unknown, that is, $\mathrm{r}$ :

$$
3 r^{2}-\frac{3 r H_{R}}{\cos \theta \tan \beta}+\left[\left(\frac{H_{R}^{2}}{\cos ^{2} \theta \tan ^{2} \beta}\right)-\left(\frac{3 V}{n H_{R} \sin \theta \cos \theta}\right)\right]=0 .
$$

This last equation yields $\mathrm{r}$ for a truncated right regular pyramid given its independent variables $\mathrm{V}, \theta, \beta$ and $\mathrm{H}_{\mathrm{R}}$. Given $\mathrm{r}$, the other variables of such pyramid can be calculated. As a quadratic equation, its three constants $\mathrm{a}, \mathrm{b}$ and $\mathrm{c}$ can be calculated as:

$$
\begin{gathered}
a=3 ; \\
b=-\frac{3 H_{R}}{\cos \theta \tan \beta} ; \\
c=\frac{H_{R}^{2}}{\cos ^{2} \theta \tan ^{2} \beta}-\frac{3 V}{n H_{R} \sin \theta \cos \theta} .
\end{gathered}
$$

In such a pyramid, there are three possibilities for modifying its dimensions, assuming that $\mathrm{V}$ is constant:

1. $\quad \beta$ and Ar are variables whereas $\mathrm{n}$ (thus $\theta$ ) is constant.

2. $\quad \beta$ is constant whereas $n$ and $\operatorname{Ar}$ (i.e., r) are variables. 


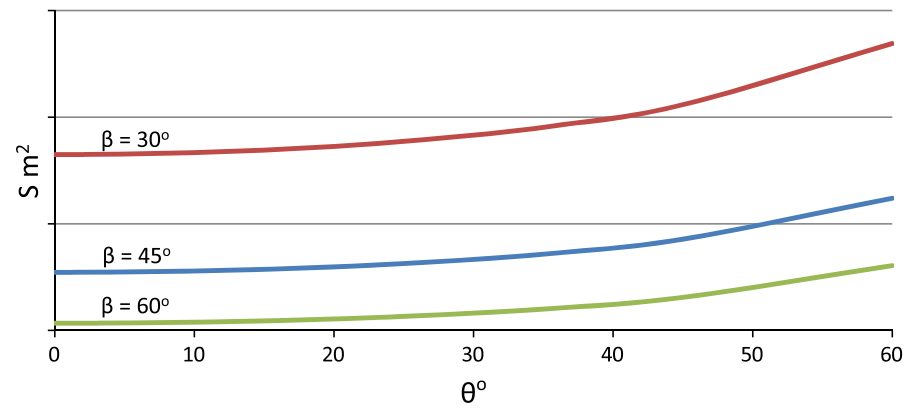

Fig. 8 The relationship of $\theta$ to $S$, different cases of $\beta$

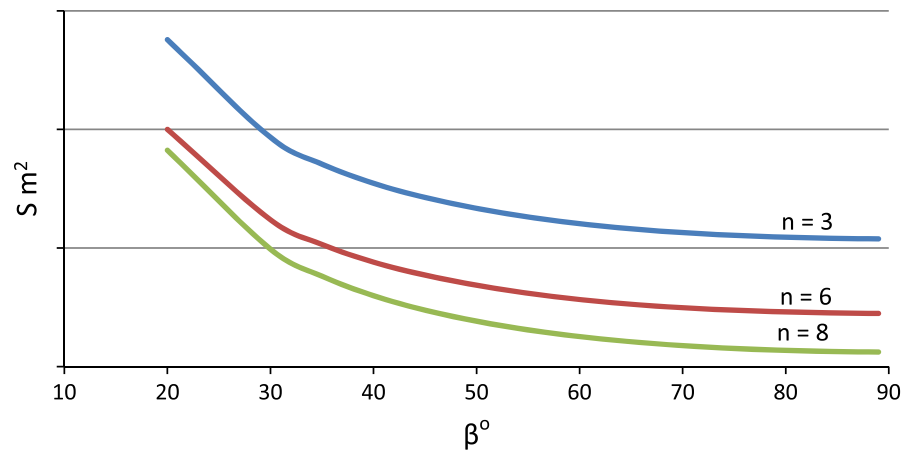

Fig. 9 The relationship of $\beta$ to $S$, different cases of (n)

3. $\mathrm{H}_{\mathrm{R}}$ is variable whereas both $\beta$ and $\theta$ are constants.

Again, in all cases Ar is variable as long as $\mathrm{V}, \beta$ and $\mathrm{H}_{\mathrm{R}}$ were determined.

Remark 1: Effects of $\theta, \beta$ and $\mathrm{H}_{\mathrm{R}}$ on $\mathrm{S}$

In a right regular truncated pyramid that has a given $\mathrm{V}$, the numerical solutions for Eqs. 25 and 29 indicate that:

- $\quad S$ is an increasing function of $\theta$ when $H_{R}$ and $\beta$ are constants. Thus $S_{\text {Min }}$ occurs when $\theta \rightarrow 0$ (i.e., a cone). Figure 8 represents $\theta-S$ relationship for different cases of $\beta$.

- $\quad \mathrm{S}$ is a decreasing function of $\beta$ when $\mathrm{H}_{\mathrm{R}}$ and $\theta$ are constants. Thus $\mathrm{S}_{\text {Min }}$ occurs when $\beta \rightarrow 90$ (i.e., a prism). Figure 9 represents $\beta-S$ relationship for different cases of (n).

- $\quad \mathrm{S}$ is a decreasing function of $\mathrm{H}_{\mathrm{R}}$ when $\theta$ and $\beta$ are constants, thus $\mathrm{S}_{\text {Min }}$ occurs when $\mathrm{H}_{R}$ is maximum. Figure 10 represents $\mathrm{H}_{\mathrm{R}}-\mathrm{S}$ relationship for different cases of $\theta$ and $\beta$. 


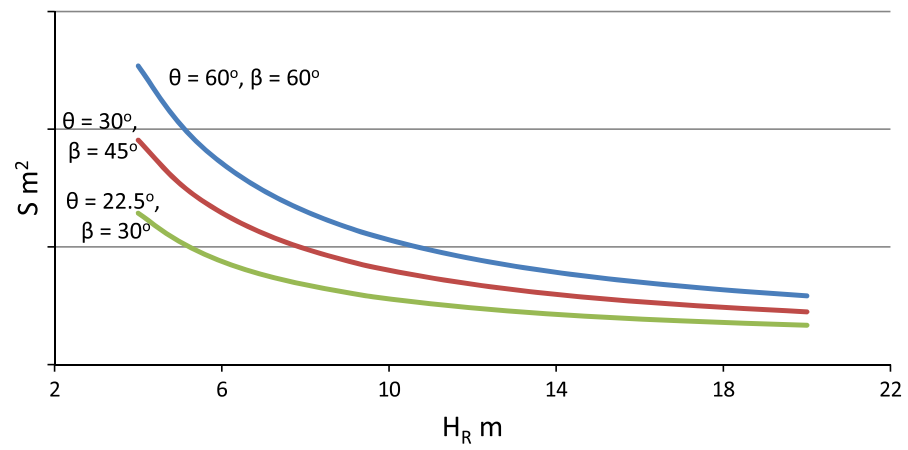

Fig. 10 The relationship of $H_{R}$ to $S$, different cases of $\theta$ and $\beta$

Remark 2: Walls Ratio $\mathrm{R}_{\mathrm{W}}$

In a right regular truncated pyramid, $\mathrm{R}_{\mathrm{W}}$ can be mathematically calculated as:

$$
R_{W}=\frac{n h_{S}\left(\frac{L+L_{U}}{2}\right)}{n h_{S}\left(\frac{L+L_{U}}{2}\right)+A r+A r_{U}} .
$$

Given the equivalent values of $\mathrm{h}_{\mathrm{S}}, \mathrm{L}, \mathrm{L}_{\mathrm{U}}$ and $\mathrm{Ar}$ (see Part III), Eq. 33 can be rewritten as:

$$
R_{W}=\frac{H_{R} \sin \theta\left(r+r_{U}\right)}{H_{R} \sin \theta\left(r+r_{U}\right)+\cos \theta \sin \theta \sin \beta\left(r^{2}+r_{U}^{2}\right)} .
$$

If $\mathrm{H}_{\mathrm{R}}$ was replaced by its equivalent value according to Eqs. 22 and 23, then Eq. 34 will be:

$$
R_{W}=\frac{r^{2}-r_{U}^{2}}{\left(r^{2}-r_{U}^{2}\right)+\cos \beta\left(r^{2}+r_{U}^{2}\right)} .
$$

Similar to the case of the complete pyramid, Eq. 35 says that $\mathrm{R}_{\mathrm{W}}$ is independent on both $\mathrm{V}$ and $\theta$, but it is a function of $\mathrm{r}, \mathrm{r}_{\mathrm{U}}$ and $\beta$. It is clear that $\boldsymbol{R}_{\boldsymbol{W o}}$ will never exist as long as the three functions $\left(\theta-S, \beta-S\right.$ and $\left.H_{R}-S\right)$ remain constant in direction.

\section{Remark 3: Case of Numerical Equality}

As mentioned above, the condition for the numerical equality between Ar and Per is the same as the case of regular multi-sided right prisms. Thus, the following section considers only the case of numerical equality between $\mathrm{S}$ and $\mathrm{V}$.

\section{Equality of $S$ and $V$}

According to Eqs. 25 and 27, this numerical equality occurs when:

$$
n h_{S}\left(\frac{L+L_{U}}{2}\right)+A r+A r_{U}=\frac{1}{3} n H_{R o} \sin \theta \cos \theta\left(r^{2}+r_{U}^{2}+r . r_{U}\right) .
$$


By replacing $\mathrm{h}_{\mathrm{S}}, \mathrm{L}, \mathrm{L}_{\mathrm{U}}, \mathrm{Ar}$ and $\mathrm{Ar}_{\mathrm{U}}$ with their equivalent values, Eq. 36 will be:

$H_{R o} \cos \theta \sin \beta\left(r^{2}+r_{U}^{2}+r . r_{U}\right)-3 H_{R o}\left(r+r_{U}\right)-3 \cos \theta \sin \beta\left(r^{2}+r_{U}^{2}\right)=0$.

By substitution for $\mathrm{r}_{\mathrm{U}}$ according to Eq. 24, Eq. 37 can be rewritten as:

$$
\begin{aligned}
& H_{R o}^{3}\left(\frac{\sin \beta}{\cos \theta \tan ^{2} \beta}\right)-H_{R o}^{2}\left(\frac{3 r \sin \beta}{\tan \beta}+\frac{3 \sin \beta}{\cos \theta \tan ^{2} \beta}-\frac{3}{\cos \theta \tan \beta}\right) \\
& \quad+H_{R o}\left(\frac{6 r \sin \beta}{\tan \beta}+3 r^{2} \cos \theta \sin \beta-6 r\right)-6 r^{2} \cos \theta \sin \beta .
\end{aligned}
$$

This last formula calculates the critical height $\boldsymbol{H}_{\boldsymbol{R}}$ that fulfills the equality between $\mathrm{S}$ and $\mathrm{V}$ in a right regular truncated pyramid when its three variables $\mathrm{Ar}$ (i.e., r), n (i.e., $\theta$ ) and $\beta$ are known. It is a cubic equation that can be solved manually or utilizing one of the free software programs available on the Internet (see for example: http://www.1728.org/cubic.htm). As a cubic equation, its four constants a, b, c and d can be calculated as:

$$
\begin{gathered}
a=\frac{\sin \beta}{\cos \theta \tan ^{2} \beta} \\
b=-\left(\frac{3 r \sin \beta}{\tan \beta}+\frac{3 \sin \beta}{\cos \theta \tan ^{2} \beta}-\frac{3}{\cos \theta \tan \beta}\right) \\
c=\frac{6 r \sin \beta}{\tan \beta}+3 r^{2} \cos \theta \sin \beta-6 r \\
d=-6 r^{2} \cos \theta \sin \beta .
\end{gathered}
$$

Given the value of $\boldsymbol{H}_{\boldsymbol{R} \boldsymbol{o}}, \mathrm{r}_{\mathrm{U}}$ can be calculated by applying Eq. 24, consequently the other parameters of such pyramid can be calculated. In this context, it is important to mention that this equality will never exist if:

$$
\frac{H_{R}}{\cos \theta \tan \beta}>r \text {. }
$$

\section{Discussion}

All is number, said Pythagoras (580-500 BC) more than twenty centuries ago. Thus, everything finally returns to mathematics. Numbers always give a better understanding for the behavior of a phenomenon as it appears. They also provide a scientific basis for predicting the ways in which things behave. The findings of this work have important applications in different disciplines of advanced building analysis, especially room acoustics as will be discussed in the following section. In summary, these findings:

- point out special rooms that have a distinct characteristic in acoustics;

- clarify why rooms that have the same volume and floor area but different shapes have different reverberation times; 
- establish a simple mathematical approach that can help both architects and acousticians to decide early the appropriate room dimensions. These dimensions satisfy the acoustic requirements;

- help architects and acousticians to answer two important questions:

- how does $\mathrm{S}$ changes in $\theta$ (and/or $\beta$ ) and consequently how will the acoustic environment within a room be affected?

- When deciding upon the appropriate room dimensions that have a given $\mathrm{V}$, is it better to decrease $\mathrm{Ar}$ and increase $\mathrm{H}_{\mathrm{R}}$ or inversely, to increase $\mathrm{Ar}$ and decrease $\mathrm{H}_{\mathrm{R}}$ ?

The reverberation time $\mathrm{T}$ (the persistence of sound in a particular space after the original sound is produced) is a main indicator in room acoustics. In its simplest form, $\mathrm{T}$ can be calculated as:

$$
T=\frac{0.161 \times V}{\sum_{i=1}^{n} S_{i} \times \alpha_{i}}(s)
$$

(Sabine 1993; Cox and D'Antonio 2009), where $\alpha$ is a physical quantity that expresses the ability of a material to absorb the energy of sound.

Upon designing a room acoustically, its $\mathrm{T}$ must lay within the permissible limits that depend on the acoustic function of this room. A short $T$ (around $1 \mathrm{~s}$ ) is recommended especially in speech rooms. As can be concluded from Eq. 44, for a given $\mathrm{V}, \mathrm{T}$ is a decreasing function of $\mathrm{S}$. Thus, it is recommended to increase room total surface area so as to decrease $\mathrm{T}$.

Based on the findings of this work and according to the shape, remark (1) determines the conditions under which $S$ will take its minimum value among the different rooms that have the same floor area and volume but different $\theta$ (and/or $\beta$ ). For example, in rectangular right prisms (or rooms), a room that has $\theta=45^{\circ}$ (square plan) has the minimum total surface area, thus the maximum $\mathrm{T}$ among the other rectangular rooms that have the same volume assuming that $\alpha$ is constant (Elkhateeb 2012).

Following the same rules, remark (2) determines the condition under which $\mathrm{S}$ will be minimum among the different rooms that have the same $\theta$ (and/or $\beta$ ) and $\mathrm{V}$ but different Ar. Such a room also possesses the maximum $\mathrm{T}$ and should be avoided (Elkhateeb 2012). Together, remarks (1) and (2) establish a clear methodology that can be applied to select the optimum room dimensions from an acoustic point of view.

In any room, walls are the typical place to install the different acoustic treatments such as absorbing and reflecting materials. Acoustically, in some applications, it is preferable to increase walls ratio so as to insure a good acoustic performance. Utilizing remark (3), $\mathrm{R}_{\mathrm{W}}$ can be checked easily during the analysis and design phase.

The shape factor $\boldsymbol{S} \boldsymbol{h}_{\boldsymbol{f}}$ is a mathematical indicator that has a direct effect on room acoustics (Elkhateeb 2012). This indicator can be calculated from:

$$
S h_{f}=\frac{P e r}{A r} \times D i s_{f},
$$

where $D i s_{f}$ is the distortion factor that is the ratio between the total surface area S1 of the examined room and the total surface area $\mathrm{S}$ of the reference room RR (i.e., 

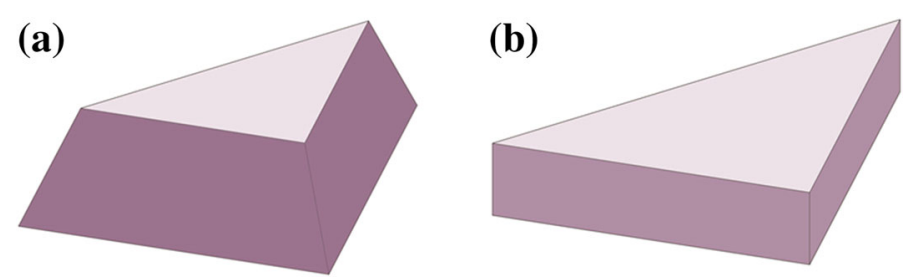

Fig. 11 The concept of reference room (RR), Dis $s_{f}=\mathrm{S} 1 / \mathrm{S}$. a left Examined room (S1); b right $\mathrm{RR}(\mathrm{S})$

$\left.D i s_{f}=\mathrm{S} 1 / \mathrm{S}\right)$. The reference room is a right prismatic room that has the identical base, floor area and volume of the examined room (Fig. 11). The indicator $\boldsymbol{S h}_{\boldsymbol{f}}$ has been suggested as a simple tool that can be used to compare architecturally, on acoustic bases, between rooms that have the same floor area but different shapes. It is preferable to choose the room that has the maximum $\boldsymbol{S} \boldsymbol{h}_{\boldsymbol{f}}$. When the perimeter of a given shape equals its area according to remark (4), accordingly and under the assumed conditions, $\boldsymbol{S} \boldsymbol{h}_{\boldsymbol{f}}=1$. In this case, $\boldsymbol{S} \boldsymbol{h}_{\boldsymbol{f}}$ can't be used as a comparison tool, and more advanced acoustic analysis will be essential.

The mean free path $l$ (the average distance travelled by sound ray between successive collisions) is another important acoustic indicator. Under certain conditions $l$ can be calculated from:

$$
l=\frac{4 V}{S}(m)
$$

(Kuttruff 2009). According to remark (4), when $\mathrm{S}$ equals $\mathrm{V}$, thus, $l$ will be equal to $4 \mathrm{~m}$. In this specific case, $\mathrm{T}$ will be equal to $(\mathbf{0 . 1 6 1 / \alpha})$ if $\alpha$ is constant for all boundaries of the room. In this last case, if $\alpha=1$ (i.e., a perfect absorber), $\mathrm{T}$ will be equal to $0.161 \mathrm{~s}$, regardless of the values of both $\mathrm{S}$ and $\mathrm{V}$, as long as they are equal.

\section{Conclusions}

Applying the same methodology, assumptions and rules that were introduced in the previous Parts I-III of this work, this final Part IV examines the case of the right regular pyramid either complete or truncated. In complete pyramids, the first remark examines the effects of $\theta$ and $\beta$ on $\mathrm{S}$. In the second remark, the minimum total surface area $S_{\text {Min }}$ was calculated in two cases, case of variable $\beta$ and constant $\theta$, and case of constant $\beta$ and variable $\theta$. In the first case, the critical ratio $\omega_{o}$ that corresponds to the critical dihedral angle $\boldsymbol{\beta}_{\boldsymbol{o}}\left(70.528779^{\circ}\right)$ was calculated. Results showed that $\omega_{o}$ depends entirely on $\theta$. The values of $\omega_{o}$ were calculated and presented for the common right regular pyramids according to their bases. In the second case, where $\theta$ is variable, results showed that $S_{\text {Min }}$ occurs when $\theta \rightarrow 0$ (i.e., cones). In the third remark, the ratio $R_{W}$ was calculated. Results showed that $R_{W}$ is an increasing function of $\beta$. Results also showed that $\boldsymbol{R}_{\boldsymbol{W o}}$ is constant $(=0.75)$ regardless the value of (n). In the last remark, the critical room height $\boldsymbol{H}_{\boldsymbol{R} o}$ that fulfills the numerical equality between $\mathrm{S}$ and $\mathrm{V}$ was calculated. Results indicated the limit under which this equality will never exist. 
In a truncated pyramid, the first remark investigates the effects of three independent variable $\theta, \beta$ and $H_{R}$ on $S$. When $\theta$ is variable (whereas $H_{R}$ and $\beta$ are constants), results showed that $S$ is an increasing function of $\theta$, thus $S_{\text {Min }}$ occurs when $\theta \rightarrow 0$ (i.e., a truncated cone). When $\beta$ is variable (whereas $H_{R}$ and $\theta$ are constants), results showed that $S$ is a decreasing function of $\beta$, thus $S_{\text {Min }}$ occurs when $\beta \rightarrow 90$ (i.e., a prism). When $\mathrm{H}_{\mathrm{R}}$ is variable (while $\theta$ and $\beta$ are constants), results showed that $S$ is a decreasing function of $H_{R}$, thus $S_{\text {Min }}$ occurs when $H_{R}$ is maximum. In the second remark, the calculation of $\mathrm{R}_{\mathrm{W}}$ indicated that it depends on the three variables $\mathrm{r}, \mathrm{r}_{\mathrm{U}}$ and $\beta$ regardless the values of $\theta$ and $\mathrm{V}$. In the last remark, the critical room height $\boldsymbol{H}_{\boldsymbol{R} \boldsymbol{o}}$ that fulfills the numerical equality between $\mathrm{S}$ and $\mathrm{V}$ was calculated. In this remark also, the limit under which this equality will never exist was presented. Finally, the importance of the findings of this entire work (Parts I-IV) in room acoustics as a branch of the advanced building analysis was presented.

Acknowledgments The author would like to express his gratitude and sincere appreciation to Editor-inChief Kim Williams for her valuable advice and support. Thanks also go to Arch Zinub Najeeb for her continuous help during the preparation of this work.

\title{
References
}

Cox, Trevor. J., and D'Antonio, Peter. 2009. Acoustic absorbers and diffusers, theory, design and application, 2nd edition. Abingdon: Spon Press.

Elkhateeb, Ahmed A. 2012. Form-acoustics relationship, effect of room shape and volume on the quality of speech. Germany: Lambert Academic Publishing.

Elkhateeb, Ahmed A. 2014a. Remarks on the surface area and equality conditions in regular forms part I: triangular prisms. Nexus Network Journal 16(1): 219-232.

Elkhateeb, Ahmed A. 2014b. Remarks on the surface area and equality conditions in regular forms part III: multi-sided prisms. Nexus Network Journal 16(2): 487-500.

Elkhateeb, Ahmed A., and Esraa A. Elkhateeb. 2014. Remarks on the surface area and equality conditions in regular forms part II: quadratic prisms. Nexus Network Journal 16(2): 467-485.

Gieck, Kurt, and Reiner Gieck. 2006. Engineering Formulas, 8th ed. Germany: McGraw-Hill.

Kuttruff, Heinrich. 2009. Room Acoustics, 5th ed. Abingdon: Spon Press.

Sabine, Wallace. 1993. Collected Papers on Acoustics. Los Altos: Peninsula Publishing.

\begin{abstract}
Ahmed A. Elkhateeb is an Egyptian architect who graduated in 1990 from the Department of Architecture, Faculty of Engineering of Ain Shams University (Cairo, Egypt). He worked in the same department until summer 2011. He completed his M.Sc. and Ph.D. in the field of architectural acoustics, which is his primary area of interest along with mathematics and its relation with architecture. He has many published research works in national and international scholarly journals in the field of building science, in addition to five published books in the same research area. He also supervised many theses in different areas related to architecture. He was awarded two international research Grants in the field of architectural acoustics. As of fall 2012 he is Full Professor of Architecture and Building Science in the Department of Architecture in the Faculty of Environmental Designs of King Abdulaziz University, Jeddah, Saudi Arabia.
\end{abstract}

Esraa A. Elkhateeb is an Egyptian Scientist graduated in 1986 from the Department of Physics, Faculty of Science of Ain Shams University (Cairo, Egypt). She worked in the same department. She completed her M.Sc. and Ph.D. in the field of theoretical physics. She has many published researches in national and international journals in Atomic, molecular, computational and high energy physics. 